\title{
Alteration of shock aversion thresholds during the menstrual cycle
}

\author{
W. H. TEDFORD, JR., D. E. WARREN, and W. E. FLYNN \\ Southern Methodist University, Dallas, Texas 75275
}

\begin{abstract}
Aversion thresholds for electrical shock were obtained from 5 males, 6 females who were taking oral contraceptives, and 12 females who were not. All subjects were volunteers from introductorylevel psychology courses. Measures were repeated 3 times per week for 5 weeks. Males and females taking oral contraceptives showed no cyclic fluctuations in threshold. The other female group had significant $(\mathrm{p}<.025)$ fluctuations in threshold, from a maximum at ovulation to a minimum 1 week after the onset of the menses.
\end{abstract}

The experience of premenstrual and menstrual discomfort is one of the most obvious and common of cyclic behavioral changes. One group of researchers (Morton, Additon, Addison, Hunt, \& Sullivan, 1953) found, in response to a questionnaire given to 600 female prison inmates, that $70 \%$ of those questioned experienced premenstrual low abdominal pain. Considering the numerous menstrually related sensory changes found in most of the other modalities, a shift in some sensory threshold may provide a partial explanation for premenstrual and menstrual discomfort. Using the common example of low back pain, it is conceivable that no new aversive stimulus is responsible for the reported discomfort. Rather the pain or pressure threshold may decrease sufficiently so that normally subthreshold stimuli become quite detectable, to the extent of becoming aversive. The concept of a cyclic lowering of a sensory threshold which could evoke menstrual discomfort is given credence by the research of Haman (1944), who tested a similar hypothesis. He said, "The question arises as to whether dysmenorrheic patients actually receive more intense pain stimuli than do nondysmenorrheic, or whether they are merely more sensitive to pain than their more fortunate sisters" (p. 686). (Dysmenorrhea refers to the syndrome of those women who report an unusually severe degree of pain accompanying menstruation.) He measured the pain thresholds of 100 dysmenorrheic women, 100 nondysmenorrheic women, 100 postmenopausal women, and 100 men, testing each person only once. A sensimeter was used to measure pain thresholds. The sensimeter measures the amount of pressure being exerted upon a protruding semi-blunt joint. This point was placed on the proximal phalanx of the thumb. The experimenter then exerted pressure until the person winced or drew away. It was the job of the experimenter to detect such a response and to read off, at that instant, the amount of pressure exerted as indicated by the needle on the dial. This device has obvious deficiencies. Nevertheless,
Haman found that dysmenorrheic women had a significantly lower pain threshold than did nondysmenorrheic women, postmenopausal women, or men. There were no significant differences between the other three groups. These results support the concept that normal menstrual discomfort may be a function of a cyclic lowering of the pain threshold.

Herren (1933) investigated cyclic changes in the two-point threshold for both pressure and pain. He divided the menstrual cycle into three phases (premenstrual, intermenstrual, and postmenstrual), taking threshold measurements once per phase in each cycle for each of five women through 11 complete cycles. He found a significant premenstrual increase in pain and pressure sensitivity with no significant differences between the intermenstrual and postmenstrual phases. Many other types of cyclic sensitivity changes have been reported (e.g., Silverman, Buchsbaum, \& Henkin, 1969).

The present study was designed to investigate cyclic changes in the aversion threshold for electrical shock, using a response-adjusting titration procedure developed by Weiss and Laties (1958).

\section{METHOD}

\begin{abstract}
Subjects
There were originally 36 volunteers for the study, 28 females and 8 males. In the course of the experiment, 8 of the females and 3 of the males either dropped out or were eliminated from the study by the experimenters due to excessive or untimely absences. Subjects were asked if absences were due to menstrual discomfort and none reported any connection. The data from 2 of the remaining 20 females were eliminated from all statistical consideration due to a failure to menstruate in one case and weekly menstruation in the other. The length of the menstrual cycles of the remaining 18 females ranged from 26 to 35 days. In response to a pretest questionnaire, 6 of these females reported that they were taking oral contraceptives. All of the volunteers were enrolled in introductory-level psychology classes. All reported good health and none reported taking any medication, other than birth control pills, which could be expected to have an effect upon the experimental measures. Subjects were asked
\end{abstract}


to record the first day of menstrual flow if it occurred during the experiment.

\begin{abstract}
Apparatus
The apparatus in the testing room consisted of a Lehigh Valley Electronics 520-22 human test system and finger-well electrodes. These were made from $18-\mathrm{cm}$ lengths of glass tubing with an outer diameter of $3.5 \mathrm{~cm}$ and a wall thickness of $.15 \mathrm{~cm}$. Rubber stoppers were placed in one end of the two tubes. The glass portion of an eyedropper was pushed through each stopper and a wire from the shock source in the adjoining room was inserted into the tube through the eyedropper. The eyedroppers were then filled with parafin to prevent leakage. The finger wells were filled with distilled water. Programming equipment in the adjacent room consisted of electromechanical support, including a shock source meeting APA standards for human experimentation.
\end{abstract}

\section{Procedure}

The participants were tested every Monday, Wednesday, and Friday for 10 -min sessions over 5 weeks. They were told that the purpose of the task was to measure the effect of biological rhythm on perceptual vigilance and that shock was being used as a distraction. The perceptual vigilance task consisted of nine buttons, each of which was lighted for $1 \mathrm{sec}$ in a random sequence. Approximately every $5 \mathrm{sec}$, a second button was illuminated for $.3 \mathrm{sec}$ and the subject was instructed to respond within the interval by pressing one of the two illuminated .buttons. Two counters were visible to the subjects, recording correct and incorrect responses. Both failure to respond and responding at the wrong time were recorded as incorrect. Monetary prizes were awarded for the highest net scores.

The middle and index fingers of the nonpreferred hand remained in the finger wells during the entire test session. Every $2 \mathrm{sec}$, a pulse was fed to a probability randomizer; $40 \%$ of these activated an ac shock source for $.5 \mathrm{sec}$. Every $6 \mathrm{sec}$, the shock was increased by $2 \mathrm{~mW}$. The subjects were told that when the shock became uncomfortable or distracting they could "turn it off" (actually, reset a 20-step ascending shock sequence to zero) by pressing a bar. The shock level at the point where it was reset registered on a counter. The average of all resets during a session was the subject's threshold for that day.

It was felt that both the vigilance task and the random programming of the shock would serve to reduce avoidance responding. Previous experiments on the same equipment indicate that thresholds are usually somewhat higher when these are used.

\section{RESULTS}

Data from female subjects were blocked into four phases: Menstrual (Days 1-7), postmenstrual (Days 8-14), ovulatory (Days 15-21), and premenstrual (Days 21-on). For those females taking the pill, these periods were referenced to the day which menstruation would have occurred. While this introduces some uncertainty which would tend to flatten the group curve, the cycles of pill takers are usually quite regular and predictable. Male data were blocked using date of lowest threshold as the onset of menstruation. Thresholds for these blocks are shown in Figure 1.

A within-subjects analysis of variance on the data from subjects not taking oral contraceptives showed that the differences between phases were significant $(F=4.85$, df $=3,21, p<.025)$. Within-subjects analysis of the other two groups did not indicate any significant differences between phases $(F=.03$;
$F=.22$ ). Despite the appearance of the graph, between-groups differences were not significant $(\mathrm{F}=1.44, \mathrm{df}=2,15, \mathrm{p}>.25)$, although the relationship of the three groups to each other was the same as that found for the visual threshold by Diamond, Diamond, and Mast (1972). The procedure used in this experiment gives very stable measures over time for individual subjects, but differences between subjects are quite large.

The data for the 12 women who were not taking oral contraceptives were further analyzed to determine when the pain threshold changed withihn phases. This analysis was complicated by absences from testing sessions which made a direct plotting of the session averages meaningless, since the between-subject differences were large. To solve this problem, only pairs of consecutive sessions were compared at a time. The session means were used for only those women who were present in both testing sessions. The $t$ test for related measures was used to compare each set of consecutive sessions. For example, the session threshold means for the sixth and fifth sessions prior to the onset of menses were compared using data only from subjects who had been present in both sessions. Then the fifth and fourth sessions were compared, and so on, for the 12 possible comparisons. The resulting between-session differences were plotted in Figure 2 in terms of directional percentage of change relative to an overall mean aversion threshold. This plot shows that the greatest sensitivity occurred 1 week after the onset of menses and that then the threshold began a steady climb until it peaked at ovulation. A significant decrease $(t=6.34$, df $=9, p<.001)$ was found between Sessions 1 and 2 after menstruation. Significant increases $(t=3.17$ and $3.01, d f=8, p<.05)$ occurred between Sessions 6 and 7 after menstruation and between Sessions 5 and 6 before menstruation.

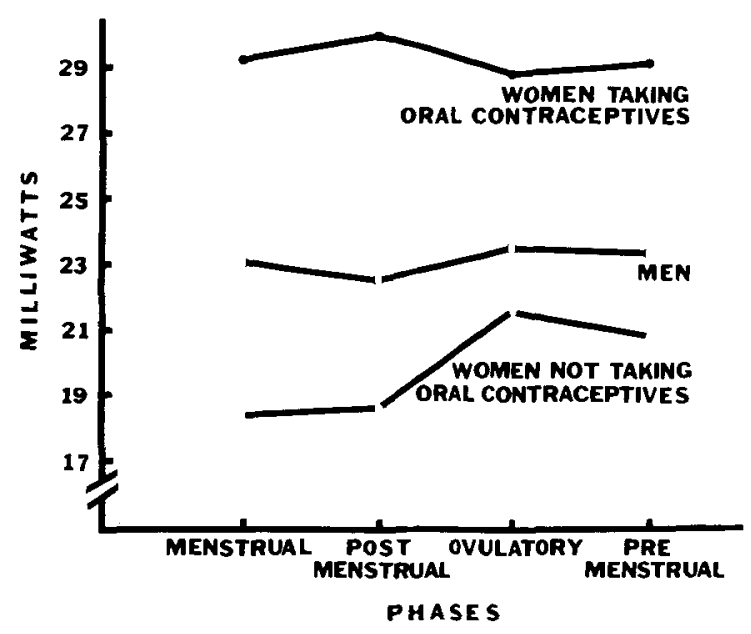

Figure 1. A verage shock threshold in each menstrual phase. 


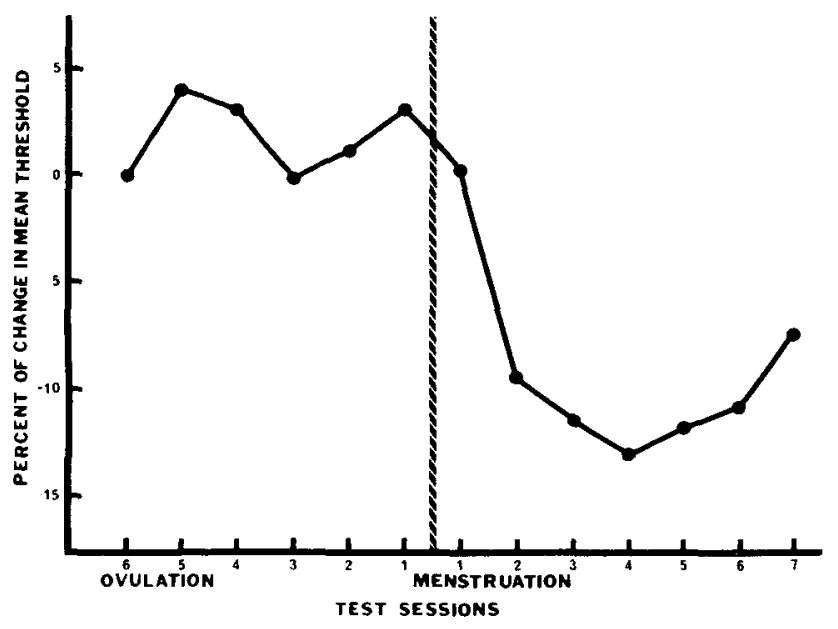

Figure 2. Changes in threshold referenced to approximate point of ovulation.

There were no systematic changes in performance on the vigilance task over the course of the experiment, except for a gradual improvement over the first few sessions. This leveled off after the third session.

\section{DISCUSSION}

The threshold measured in this study has been called "aversion" to distinguish it from traditional pain detection measures. In similar studies on the same equipment, but without the distraction task, subjects were asked to press the lever when they felt pain. Average thresholds were slightly lower, but variation between trials was much greater. It may be that when the subject focuses on the shock, the distinction between stimulus detection and pain detection becomes more blurred. Results in this laboratory have been much more stable when the distinction was made as an adjunct to another ongoing task. In the present study, most subjects reported that mild levels of shock were perceived as nonaversion tingling sensations and did not prompt lever responses.

Data from three males who volunteered to "take the shock as long as they could stand it" produced threshold values around $45 \mathrm{~mW}$. This indicates that pain tolerance was probably not being measured in the present study. However, the procedure used would not discriminate between a change in physiological sensitivity and a change in the criterion of "uncomfortable or distracting." As Clark and Goodman (1974) have shown, these may vary independently in pain detection. It might even be true that social conditioning predisposes females to expect pain during the menstrual period. But there are several possible mechanisms which might produce a physiological change in sensitivity, and these seem worth expioring.

The analysis of the data indicates that women not taking oral contraceptives had significantly lower aversion thresholds during the menstrual and postmenstrual phases. Males, and women taking oral contraceptives, exhibited no cyclic changes. It is possible that the changes in the women who were not taking the pill were due to cyclic fluctuations in the gonadal hormones, since estrogen and progesterone are artificially maintained at an approximately constant level by oral contraceptives. Klaiber, Kokayaski, Broverman, and Hall (1971) report that one of the combined effects of progesterone and estrogen fluctuations is to modify the serum and central nervous system levels of monoamineoxidase (MAO). Since MAO is well known to degrade the neurotransmitter Serotonin (Cooper, Bloom, \& Roth, 1974), and serotonin has been implicated as the neurotransmitter for pain sensitivity (Lints, 1965; Lints \& Harvey, 1969), perhaps this is the basis for threshold change.

A significant change in the aversion threshold in humans could result from physiological changes at the receptor site, central nervous system neurochemical changes, or both. A previous experiment, which prompted the present investigation, found increases in the pain threshold in rats to occur as a function of water deprivation (Flynn, Schauer, $\&$ Tedford, 1975). One other possible explanation for these results is that less extracellular fluid at the receptor site or increased skin impedance may decrease conductance into deeper tissue and thus the aversiveness of the electrical stimulation. Morton, et al. (1953) noted that the average weight increase due to premenstrual increase in water retention was about $3 \mathrm{lb}(1.36 \mathrm{~kg})$ in human females. The subsequent hypothesis evolved that a premenstrual increase in water balance could cause a decrease in the aversion threshold, which would be in line with Herren's (1933) results. However, in the present experiment, maximum sensitivity occurred approximately 1 week after maximum water retention. Whatever the causal mechanism, there seems to be a clear connection between aversion threshold and the menstrual cycle.

\section{REFERENCES}

Clark, W. C., \& Goodman, J. L. Effects of suggestion on $d^{\prime}$ and Cx for pain detection and pain tolerance. Joumal of Abnormal Psychology, 1974, 83, 364-372.

CoOper, J. R., Bloom, F. E., \& Roth, R. H. The biochemical basis of neuropharmacology. Oxford University Press: New York, 1974. Pp. 196-197.

Diamond, M., Diamond, A. L., \& Mast, M. Visual sensitivity and arousal levels during the menstrual cycle. Journal of Nervous and Mental Disease, 1972, 55. 170-175. 
Flynn, W. E., Schauer, C. A., \& Tedford, W. H., JR. Water balance and titrated pain thresholds. Psychological Reports, $1975,37,487-494$.

Haman, J. O. Pain threshold and dysmenorrhea. American Journal of Obstetrics and Gynecology, 1944, 47, 686-691.

HerRen, R. G. H. The effect of high and low female sex hormone concentration on the two-point threshold of pain and touch and upon tactile sensitivity. Journal of Experimental Psychology, $1933,16,324-327$.

Klaiber, E. L., Kobayaski, Y., Broverman, D. M., \& Hall, F. Plasma monoamine oxidase activity in regularly menstruating women and in amenorrheic women receiving cyclic treatment with estrogen and a progestrin. Journal of Clinical Endocrinology and Metabolism, 1971, 33, 630-637.

Lints, C. E. Changes in sensitivity to shock following central nervous lesions in the rat. Unpublished doctoral dissertation, University of Chicago, 1965.
Lints, C. E., \& Harvey, J. A. Altered sensitivity to foot shock and decreased content of brain serotonin following brain lesions in the rat. Journal of Comparative and Physiological Psychology, 1969, 7, 23-32.

Morton, J. H., Additon, H., Addison, R. G., Hunt, L., \& Sullivan, J. J. A clinical study of premenstrual tension. American Journal of Obstetrics and Gynecology, 1953, 65, 1182-1191.

Silverman, J., Buchsbaum, M., \& Henkin, R. Stimulus sensitivity and stimulus control. Perceptual and Motor Skills, 1969, 28, $71-78$.

Weiss, B., \& LAties, V. G. Fractional escape and avoidance on a titration schedule. Science, 1958, 128, 1575-1576.

(Received for publication August 18, 1976; revision accepted December 27,1976 .) 